\title{
IMPLEMENTATION OF THE OPEN SOURCE VIRTUALIZATION TECHNOLOGIES IN CLOUD COMPUTING
}

\author{
Mohammad Mamun Or Rashid, M. Masud Rana and Jugal Krishna Das \\ Department of Computer Science and Engineering, Jahangirnagar University Savar, \\ Dhaka, Bangladesh
}

\begin{abstract}
The "Virtualization and Cloud Computing" is a recent buzzword in the digital world. Behind this fancy poetic phrase there lies a true picture of future computing for both in technical and social perspective. Though the "Virtualization and Cloud Computing are recent but the idea of centralizing computation and storage in distributed data centres maintained by any third party companies is not new but it came in way back in 1990s along with distributed computing approaches like grid computing, Clustering and Network load Balancing. Cloud computing provide IT as a service to the users on-demand basis. This service has greater flexibility, availability, reliability and scalability with utility computing model. This new concept of computing has an immense potential in it to be used in the field of e-governance and in the overall IT development perspective in developing countries like Bangladesh.
\end{abstract}

\section{KEYWORDS}

Cloud Computing, Virtualization, Open Source Technology.

\section{INTRODUCTION}

Administrators usually use lots of servers with heavy hardware to keep their service accessible, available for the authenticated users. As days passes by concernment of new services increases which require more hardware, more effort from IT administrators. There is another issue of capacity (Hardware as well as storage and networking) which always increases day by day. Moreover sometime we need to upgrade old running servers as their resources have been occupied fully. On that case we need to buy new servers, install those services on that server and finally migrate to the service on it. Cloud computing focus on what IT always needs: a way to increase capacity on the fly without investing in new infrastructure. Cloud computing also encompasses any subscription-based, user-based, services-based or pay-per-use service that in real time over the internet extends its existing capabilities.

\subsection{Definition Of Cloud Computing}

Cloud computing is a model for enabling convenient, on-demand network access to a shared pool of configurable computing resources (e.g., networks, servers, storage, applications and services) that can be rapidly provisioned and released with minimal management effort on service provider interaction [1]. 
International Journal on Cloud Computing: Services and Architecture (IJCCSA) Vol. 6, No. 2, April 2016

\subsection{Benefits Of Cloud Computing}

Flexibility - Every day organization demands increase and Scale up or down to meet their requirements. Today's economy, flexibility is the key. One can adjust his IT expenditures to meet your organization's immediate needs.

Security - Cloud service assured that your data in the cloud is much more secure than in your small unsecured server room.

Capacity -With cloud computing, Capacity always increase and it is no longer an issue. Now, focus on how the solution will help in further mission. The IT piece belongs to somebody else.

Cost - Cloud and Virtualization technology reduce your all maintenance fees. There is no more servers, software, and update fees. Many of the hidden costs typically associated with software implementation, customization, hardware, maintenance, and training are rolled into a transparent subscription fee.

\subsection{VIRTUALIZATION}

Virtualization can be practical very broadly to just about everything you can imagine including processor, memory, networks, storage, operating systems, and applications. Three characteristics of virtualization technology make it ideal for cloud computing:

Partitioning: In virtualization technology, single physical server or system can use partitioning to support many different applications and operating systems (OS).

Isolation: In cloud computing, each virtual machine is isolated and protected from crashes or viruses in the other machines. What makes virtualization so important for the cloud is that it decouples the software from the hardware.

Encapsulation: Encapsulation can protect each application so that it doesn't interfere with other applications. By using encapsulation, each virtual machine stored as a single file, making it easy to identify and present to other applications and software. To understand how virtualization helps with cloud computing, we must understand its many forms. In all cases, a single resource actually emulates or imitates other resources. Here are some examples:

Virtual memory: Every disk has a lot more space than memory. PCs can use virtual memory to borrow extra memory from the hard disk. Although virtual disks are slower than real memory, if managed right, the substitution works surprisingly well.

Software: Virtualization software is available which can emulate an entire computer. A virtual single computer can perform as though it were actually more than computers. This kind of software might be able to move from a data centre with thousands of servers. To manage virtualization in cloud computing, most of companies are using different hypervisors. Because in cloud computing we need different operating environments, the hypervisor becomes an ideal delivery mechanism by allowing same application on lots of different systems. Hypervisors can load multiple operating systems in single node; they are a very practical way of getting things virtualized quickly and efficiently. Let's try to draw a picture on above statement. 


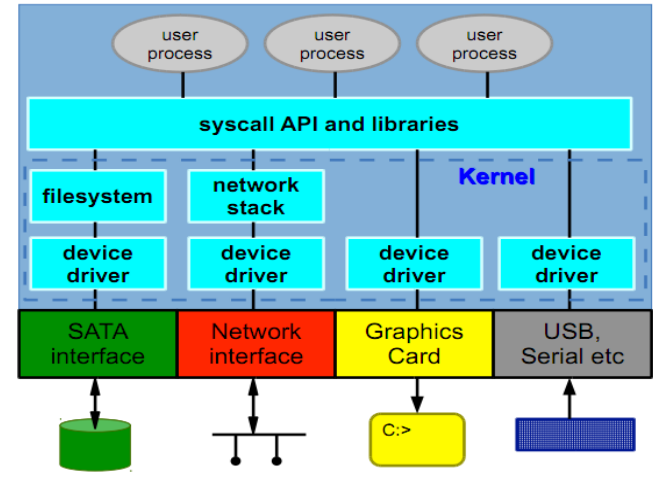

Figure 1.1: A normal Workstation / Computer

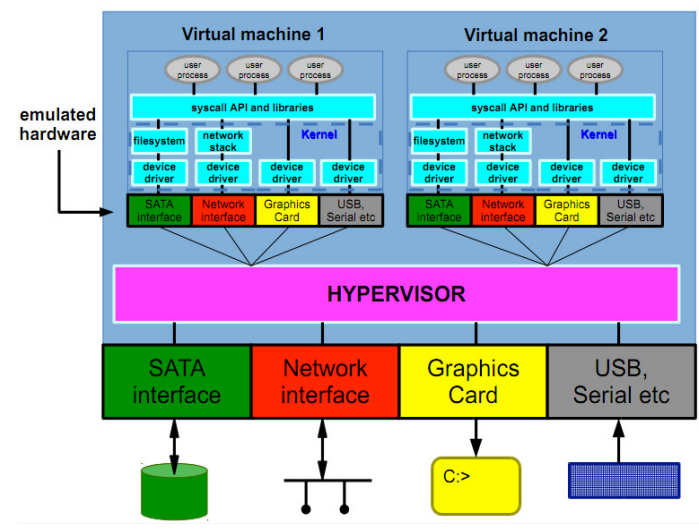

Figure 1.2: Workstation using Hypervisor

\subsection{HYPERVISOR}

The evolution of virtualization greatly revolves around one piece of very important software that loads the whole virtual system. This is the hypervisor. As an integral component of computer node, this software allows all physical devices to share their all resources (Processor, RAM, Disk, Network) amongst virtual machines running as guests on to top of that physical hardware devices.

\subsection{RELATED WORK}

Open source Virtualization technologies in Cloud computing provided this paper on multiple Node to measure its performance [2], [3], [4] and [5]. In this paper, we extend this evaluation to include Master Node as another Instance in virtualization platform, and test both under different scenarios including multiple VMs and multi-tiered systems. We have also working with oVirt in Virtualization that implemented with Centos 6. We created three Hypervisor (Node) and One Manager. There are 76 Virtual Machine running where most of them application Server and 4 Database server with Disaster Recovery System. For Application server, We have implemented NBL(Network Load Balancer) for web services to active in service 24/7. Ganeti supports a very lightweight architecture which is very useful to start with commodity hardware. From starting a single node installation an administrator can scale out the cluster very easily. It is designed to use local storage also compatible with larger storage solutions. It has fault-tolerance as a built-in feature. In a word it is very simple to manage and maintain. Ganeti is admin centric clustering solution which is the main barrier for public cloud deployment. To the best of our knowledge, 
these types of virtualization technologies have not been evaluated in the context of server clustering. Multiple Node Server consolidation using virtual containers brings new challenges and, we comprehensively evaluate two representative virtualization and cloud technologies in a number of different Node scenarios.

\section{IMPLEMENTATION}

\subsection{Scope Of This Project}

In this project we used following configuration hardware.

CPU: Dual Core

RAM: 2GB

Storage: $140 \mathrm{~GB}$

NIC: 1

We use 3 hardwires stated like above. We will use Debian GNU/Linux 7 as our base operating system and run Ganeti over the operating system using KVM as hypervisor. Later we will initiate a cluster on one physical host as a master node. We will join other nodes on that cluster. We will use a manageable switch and VLAN on it to separate our management + storage network and public facing VM network for security purpose. Later we will create VMs and check live migration, Network changes and failover scenarios.

\subsection{SumMary OF The TOPOLOGY}

We will connect three commodity computers in our cluster. Each computer has a single NIC which will be logically divided by VLANs. All the computers will be connected to a trunk port of a manageable switch to accept logical network (VLAN). The management + Storage network and pubic network (VM) will be separated from that manageable switch. The deployment architecture and physical node connectivity has been presented below.

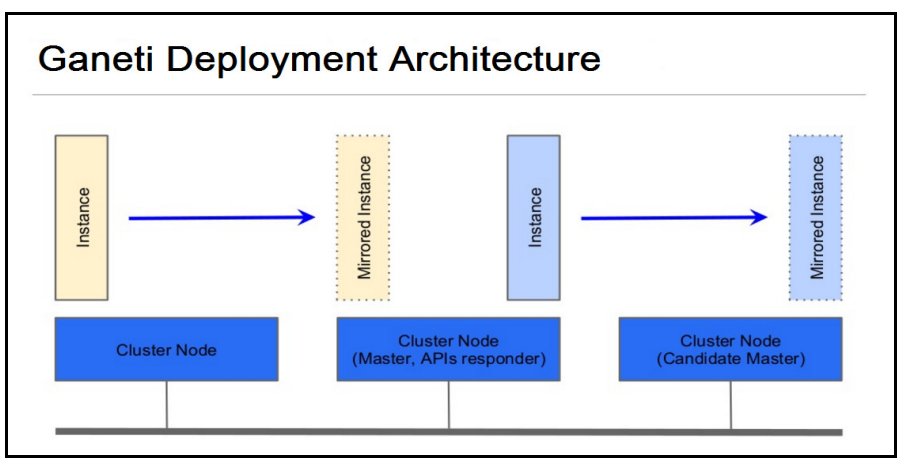

Figure2.1: Deployment Architecture 


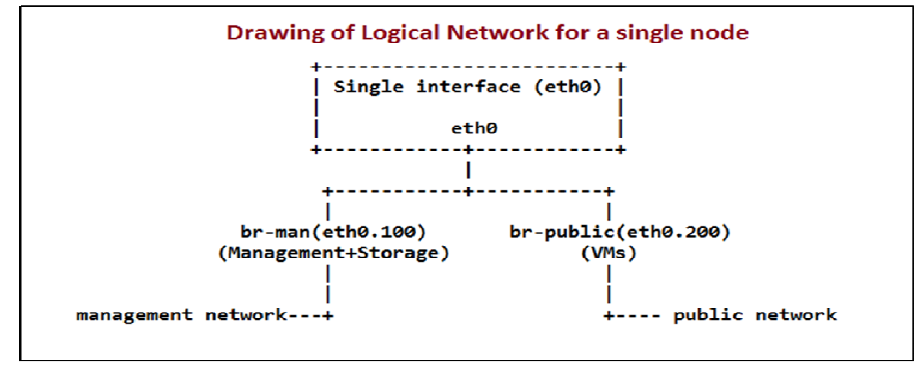

Figure 2.2: Network connectivity of a Physical Node

\subsection{InStallation Of Base OPERATing System}

This is mandatory for all nodes.

We have installed a clean, minimal operation system as standard OS. The only requirement we need to be aware of at this stage is to partition leaving enough space for a big (minimum 10GB) LVM volume group which will then host your instance file systems, if we want to use all Ganeti features. In this case we will install the base operating system on 10GB of our storage space and remaining storage space will leave un-partitioned for LVM use. The volume group name we use will be genetic.

\subsection{Configure the Hostname}

Look at the contents of the file/etc/hostname and check it contains the fully-qualified domain name, i.e. node1.project.edu

Now get the system to re-read this file:

\# hostname $-\mathrm{F}$ /etc/hostname

Also check / etc/hosts to ensure that you have the both the fully-qualified name and the short name there, pointing to the correct IP address:
127.0.0.1 localhost
192.168.20.222 node1.project.edu node1

\subsection{Creating Logical Volume Manager}

Type the following command:

\# vgs

If it shows we have a volume group called 'ganeti' then skip to the next section, "Configure the Network". If the command is not found, then install the lvm2 package:

\# apt-get install lvm2

Now, our host machine should have either a spare partition or a spare hard drive which we will use for LVM. If it's a second hard drive it will be / dev/vdb or / dev/sdb. Check which you have: 
International Journal on Cloud Computing: Services and Architecture (IJCCSA) Vol. 6, No. 2, April 2016

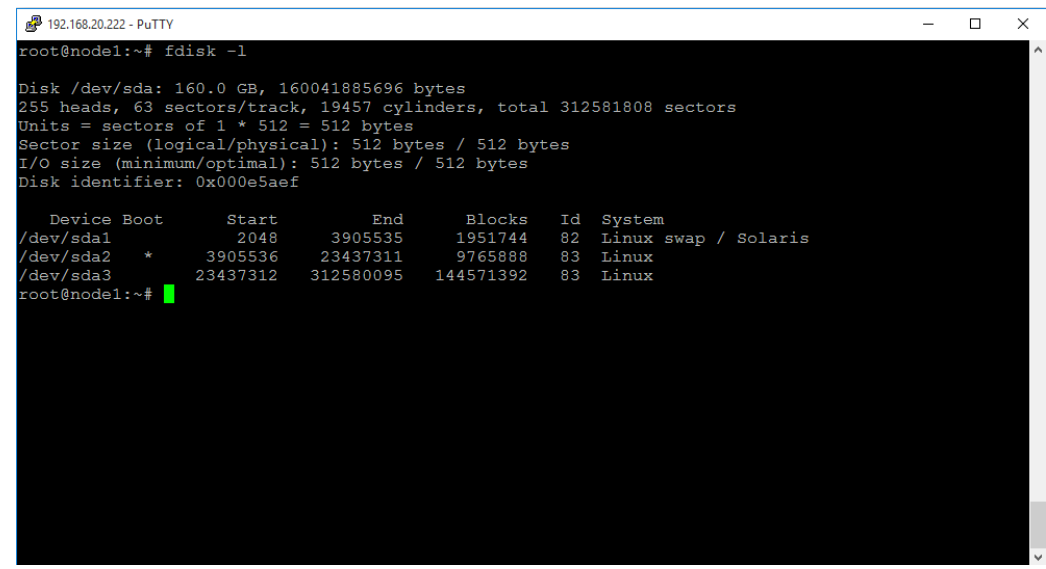

Figure 2.3: Checking available disks

Assuming / dev/sda3 is spare; let's mark it as a physical volume for LVM:

\# pvcreate / dev/sda3

\# pvs \# should show the physical volume

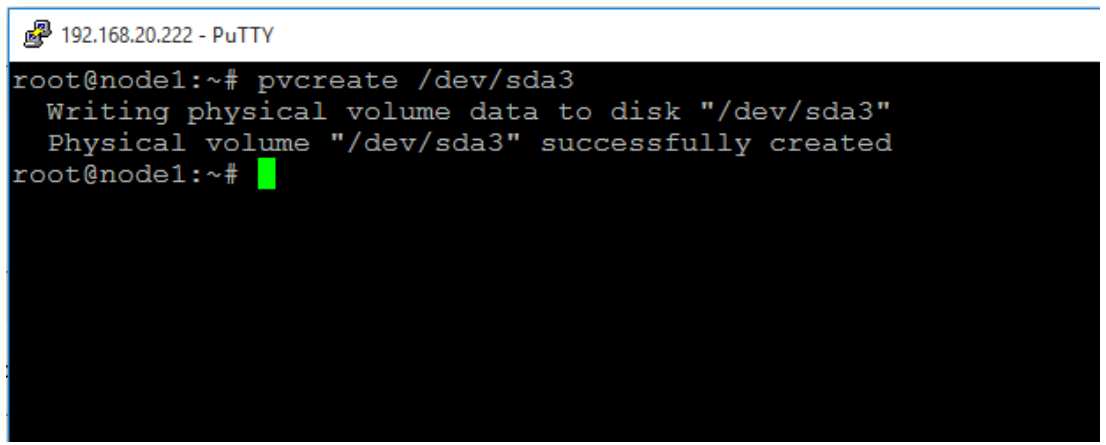

Figure 2.4: Physical Volume Create

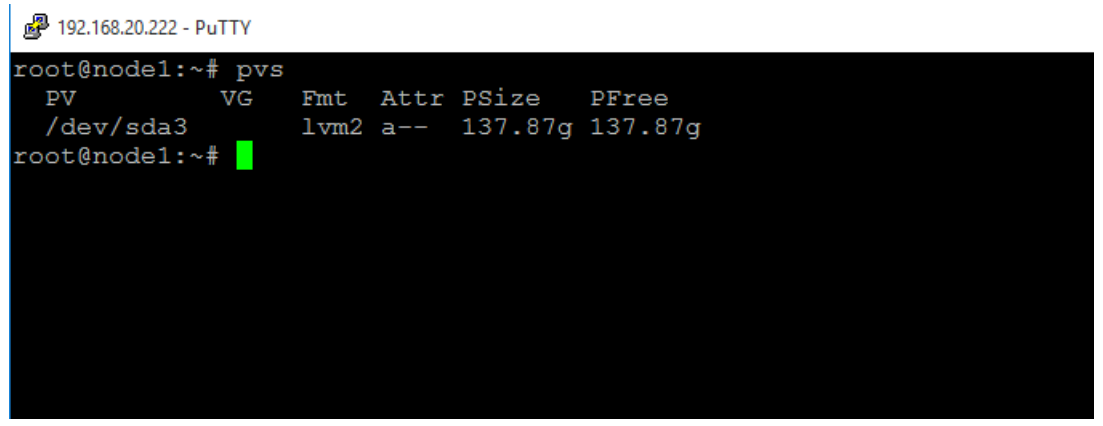

Figure 2.5: Physical volume check

Now we need to create a volume group called ganeti containing just this one physical volume. (Volume groups can be extended later by adding more physical volumes)

\# vgcreate ganeti / dev/vdb

\# vgs 


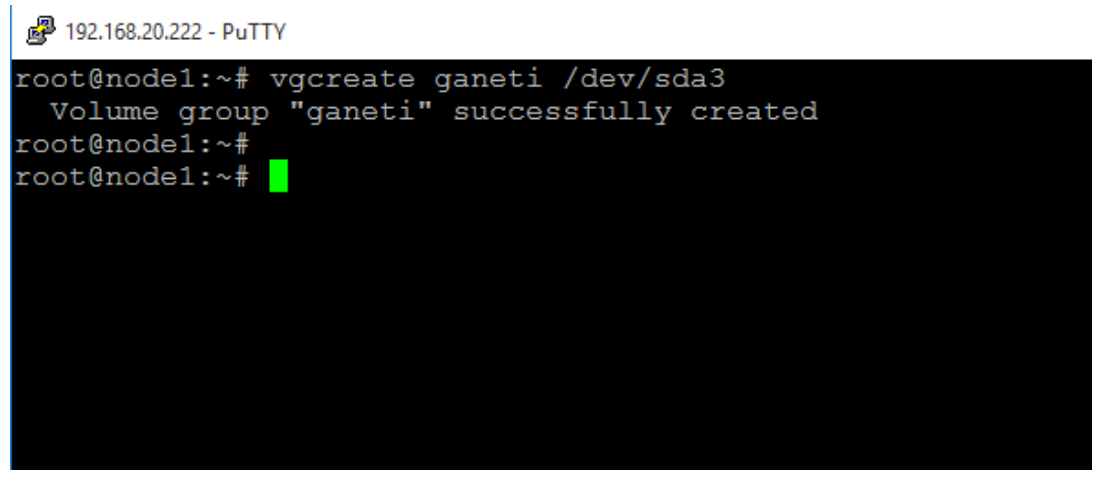

Figure 2.6: Volume Group Create

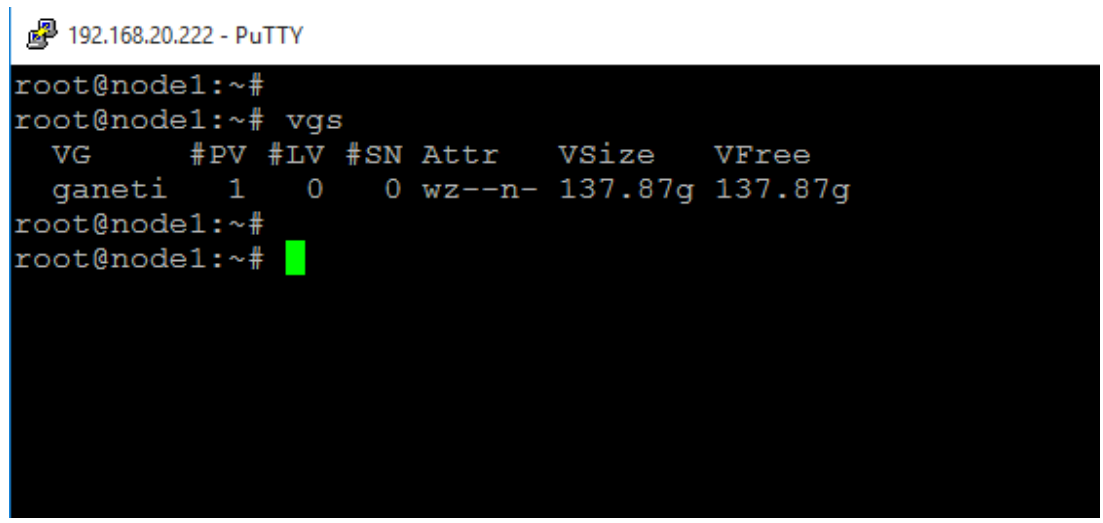

Figure 2.7: Volume Group Check

Note: on a production Ganeti server it is recommended to configure LVM not to scan DRBD devices for physical volumes. The document suggests editing / etc / l vm/lvm. conf and adding a reject expression to the filter variable, like this:

filter $=[$ "r|/dev/cdrom/", "r|/dev/drbd[0-9]+|" ]

\subsection{CONFigure The NeTWORK}

We're now going to reconfigure the network on our machine, so that we will be using VLANs. While it would be perfectly fine to use a single network for running virtual machines, there are a number of limitations, including: No separation between the networks used to manage the servers (management) and the disk replication network i.e. storage network. We will be using networkbased disk replication. We'd like to keep the disk traffic separate from the management and service traffic Instead of using separate Ethernet cards, we'll use VLANs. In commodity hardware we usually have only one network interface.

We need to implement the networks: management, replication, and service.

Ideally, we would create two VLANs:

A management + Storage VLAN (vlan 100).

An external (or service) VLAN (vlan 200), where we will "connect" the virtual machines to publish them on internet. 


\section{VLAN configuration}

To be on the safe side, let's install the vlan and bridge management tools (these should already have been installed by you earlier).

\# apt-get install vlan bridge-utils

Let's make changes to the network configuration file for your system. If you remember, this is /etc/network/interfaces.

Edit this file, and look for the br-man definition for management and storage network and brpublic for public VM network. This is the bridge interface you created earlier, and eth0 is attached to it. If should looks something like this:

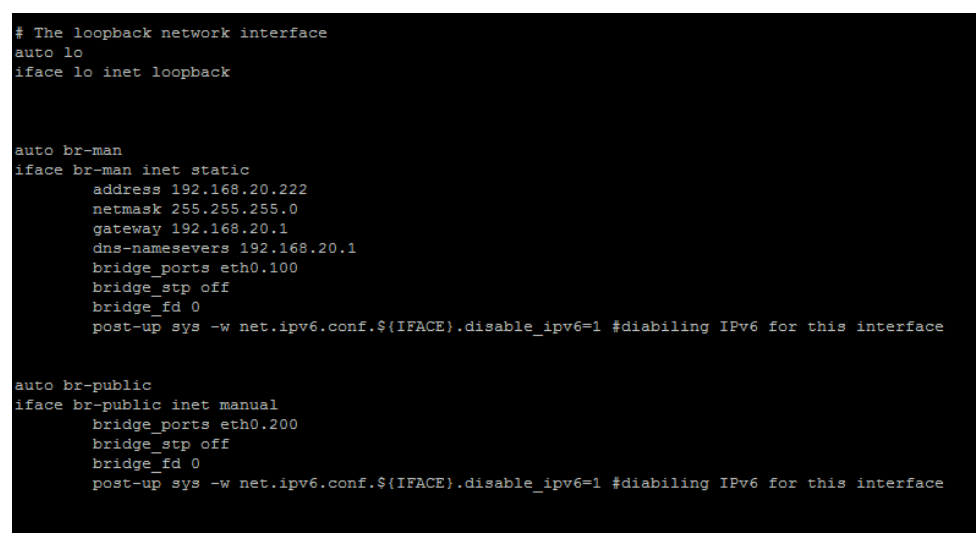

Figure 2.8: Network Interface configuration

\subsection{Synchronize The Clock}

It's important that the nodes have synchronized time, so install the NTP daemon on every node: \# apt-get install ntp

\subsection{InSTALl The Ganeti SOFTWARE}

Now install the software from the right package repository. How to do this depends on whether your machine is running Debian or Ubuntu. On Debian, the available version of ganeti is too old, but fortunately the current version is available in a back ports repository.

As root, create a file / etc/apt/sources. list.d/wheezybackports. list containing this one line: deb http://cdn.debian.net/debian/ wheezy-back ports main then refresh the index of available packages:

\# apt-get update

Now, install the Ganeti software package. Note that the back ports packages are not used unless you ask for them explicitly.

\# apt-get install ganeti/wheezy-back ports

This will install the current released version of Ganeti on our system; but any dependencies it pulls in will be the stable versions. 


\subsection{SETUP DRBD}

We'll now set up DRBD (Distributed Replicated Block Device), which will make it possible for VMs to have redundant storage across two physical machines. DRBD was already installed when we installed Ganeti, but we still need to change the configuration:

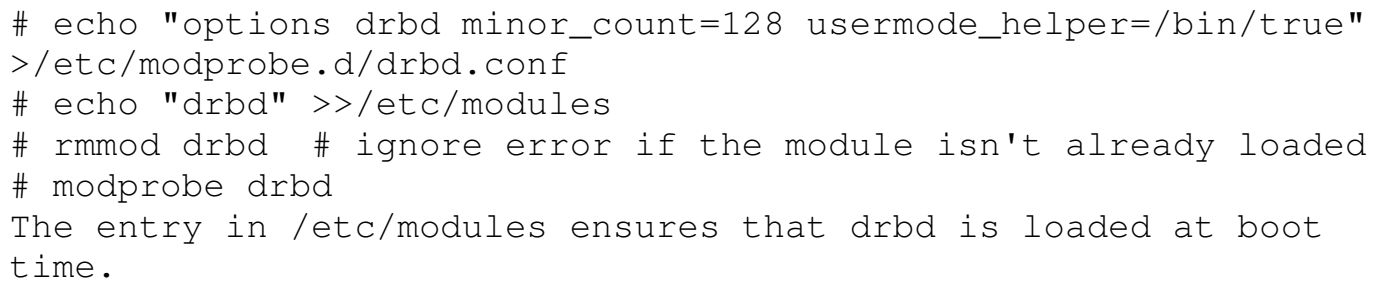

\subsection{INITIALIZE The ClUSTER - MASTER NODE ONLY}

We are now ready to run the commands that will create the Ganeti cluster. Do this only on the MASTER node of the cluster.

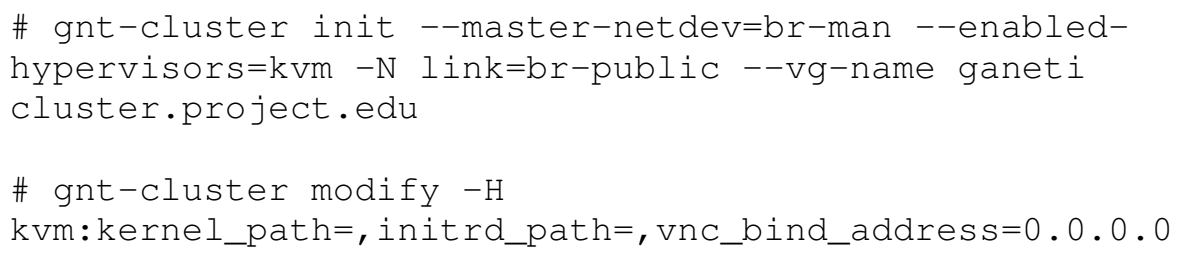

Adding nodes to the cluster - MASTER NODE ONLY

So let's run the command to add the other nodes. Note the use of the -s option to indicate which IP address will be used for disk replication on the node we are adding.

Run this command only on the MASTER node of the cluster.

\# gnt-node add node2.project.edu

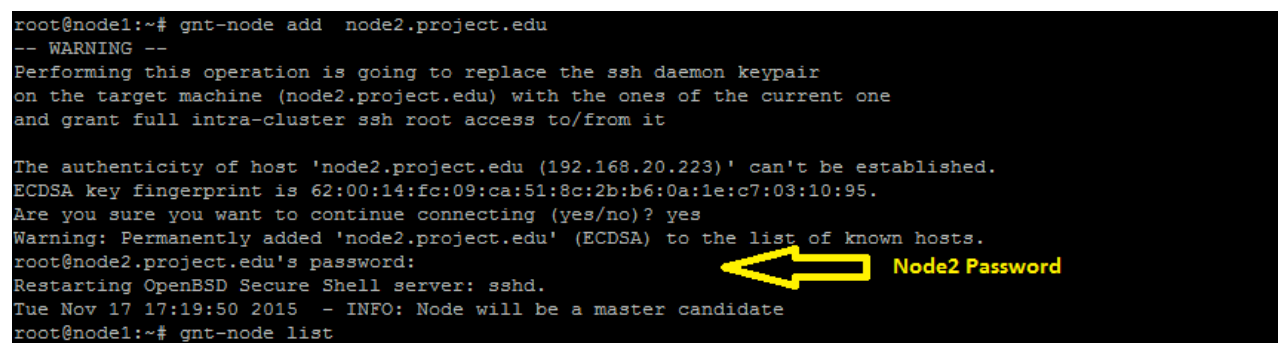

Figure 2.9: Add a node to the Cluster

We will be warned that the command will replace the SSH keys on the destination machine (the node you are adding) with new ones. This is normal.

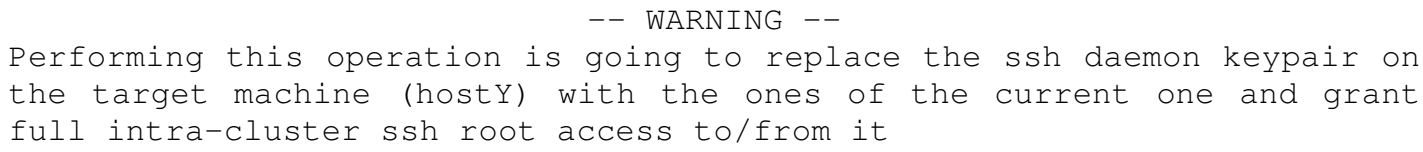


International Journal on Cloud Computing: Services and Architecture (IJCCSA) Vol. 6, No. 2, April 2016

When asked if you want to continue connection, say yes:

The authenticity of host 'node2 (192.168.20.223)' can't be established.

ECDSA key fingerprint is

a1:af:e8:20:ad:77:6f:96:4a:19:56:41:68:40:2f:06.

Are you sure you want to continue connecting (yes/no)? yes

When prompted for the root password for node2, enter it:

Warning: Permanently added 'node2' (ECDSA) to the list of known hosts.

root@node1's password:

You may see the following informational message; you can ignore it:

Restarting OpenBSD Secure Shell server: sshd.

Rather than invoking init scripts through /etc/init.d, use the service utility, e.g. service ssh restart

Since the script you are attempting to invoke has been converted to an Upstart job, you may also use the stop and then start utilities,

e.g. stop ssh ; start ssh. The restart utility is also available.

ssh stop/waiting

ssh start/running, process 2921

The last message you should see is this:

Tue Nov 17 17:19:50 2015 - INFO: Node will be a master candidate

This means that the machine you have just added into the node (hostY) can take over the role of configuration master for the cluster, should the master (hostX) crash or be unavailable.

Check the node has been added in cluster or not by following command:

\#gnt-node list

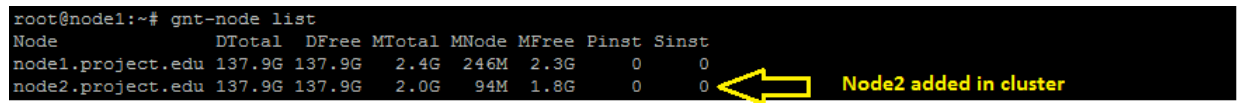

Figure 2.10: Node list

Now add the remaining node in our cluster and check the status again.

\subsection{INSTALLING OS DEFINITION - ALL NODES}

We need to install a support package called ganeti-instance-image. This provides ganeti with an "OS definition" - a collection of scripts which ganeti uses to create, export and import an operating system.

The package can be installed as follows: do this on all nodes in your cluster.

\# wget https://code.osuosl.org/attachments/download/2169/ganetiinstance-image_0.5.1-1_all.deb

\# dpkg -i ganeti-instance-image_0.5.1-1_all.deb 


\subsection{UPDATE THE OS DEFINITION - MASTER ONLY}

First wait until the other (slave) nodes in our cluster have installed the ganeti-instance-image package. Instance-image needs to be told how to install or re-install the operating system. It can be configured to do this by unpacking an image of an already-installed system (in tar, dump or qcow2 format), but in our case we just want to do a manual install from a CD image.

On the master node, as root create a file /etc/ganeti/instance-

image/variants/cd. conf with the following contents:

CDINSTALL="yes"

NOMOUNT $=$ "yes"

Aside: the full set of settings you could put in this file are listed in /etc/default/ganetiinstance-image, but don't edit them there

Now edit/etc/ganeti/instance-image/variants.list so it looks like this:

default

cd

Copy these two files to the other nodes:

\# gnt-cluster copyfile/etc/ganeti/instance-

image/variants/cd.conf

\# gnt-cluster copyfile /etc/ganeti/instance-image/variants.list

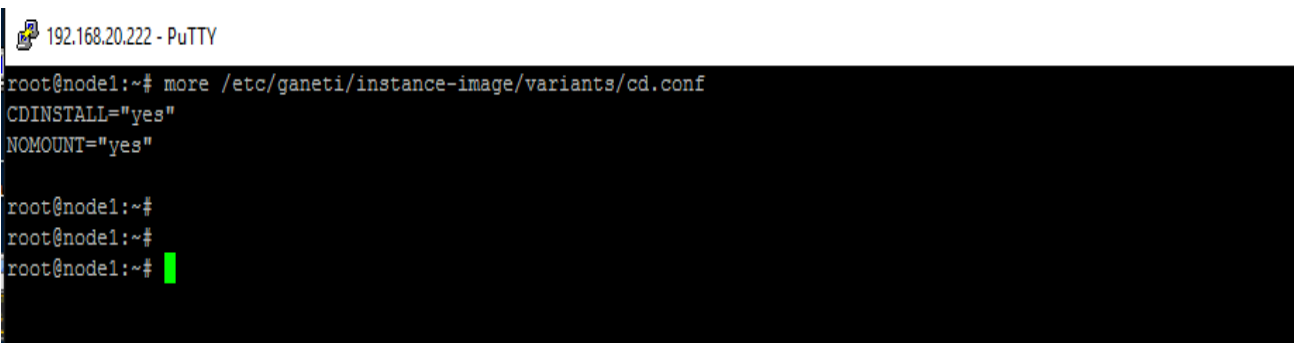

Figure 2.11: Variants Configuration

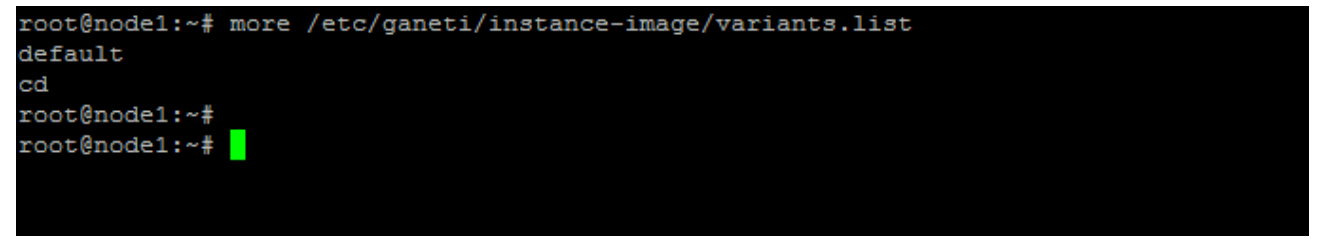

Figure 2.12: Variants check

Still on the master, check that the "image+cd" variant is available.

\# gnt-os list

Name

debootstrap+default

image+cd $\quad<$ THIS ONE

image+default 
International Journal on Cloud Computing: Services and Architecture (IJCCSA) Vol. 6, No. 2, April 2016

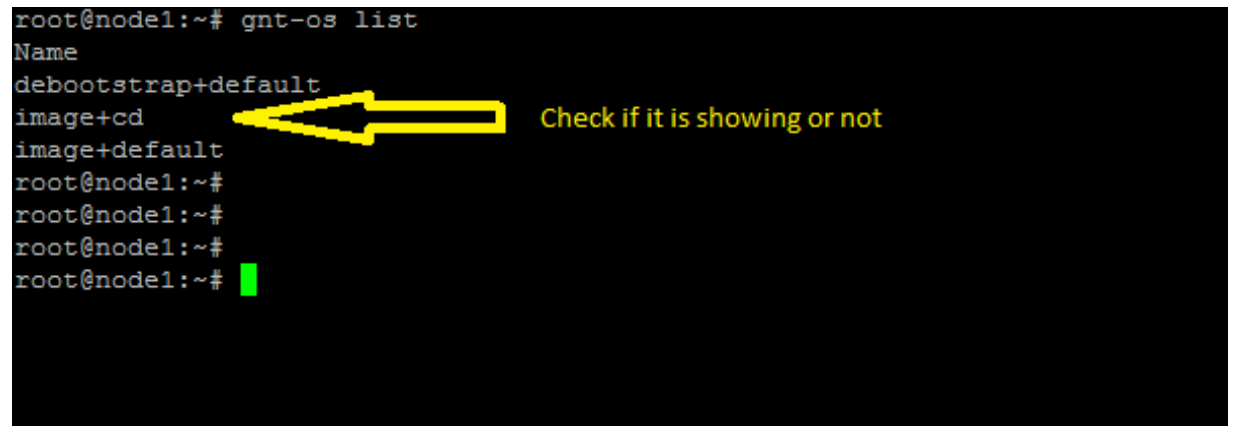

Figure 3.13: Boot source for instances

\subsection{DISTRIBUTING ISO IMAGES - ALL NODES}

If using DRBD, the ISO images used for CD installs must be present on all nodes in the cluster, in the same path. You could copy them to local storage on the master node, and then use gnt-cluster copy file to distribute them to local storage on the other nodes. However to make things simpler, we've made all the ISO images available on an NFS share (Network File Service), which you can attach. On every node, create a empty directory / i so:

\# mkdir /iso

Now copy a test OS iso in /iso directory. We have copied a debian iso image for test. Now send the iso image to every node by following command:

\# gnt-cluster copyfile /iso/debian-7.9.0-amd64-netinst.iso

\subsection{CREATION OF INSTANCE - EVERYONE ON MASTER NODE}

For example, if you working on host 3 then you will have to login to host1 (your cluster's master node). You will then create a VM called testvm.project.edu and instruct ganeti to create it on your host using the flag -n node3.project.edu. To create new an instance, run the following command. (Note that we don't start it yet, because we want to temporarily attach the CD-ROM image at start time).
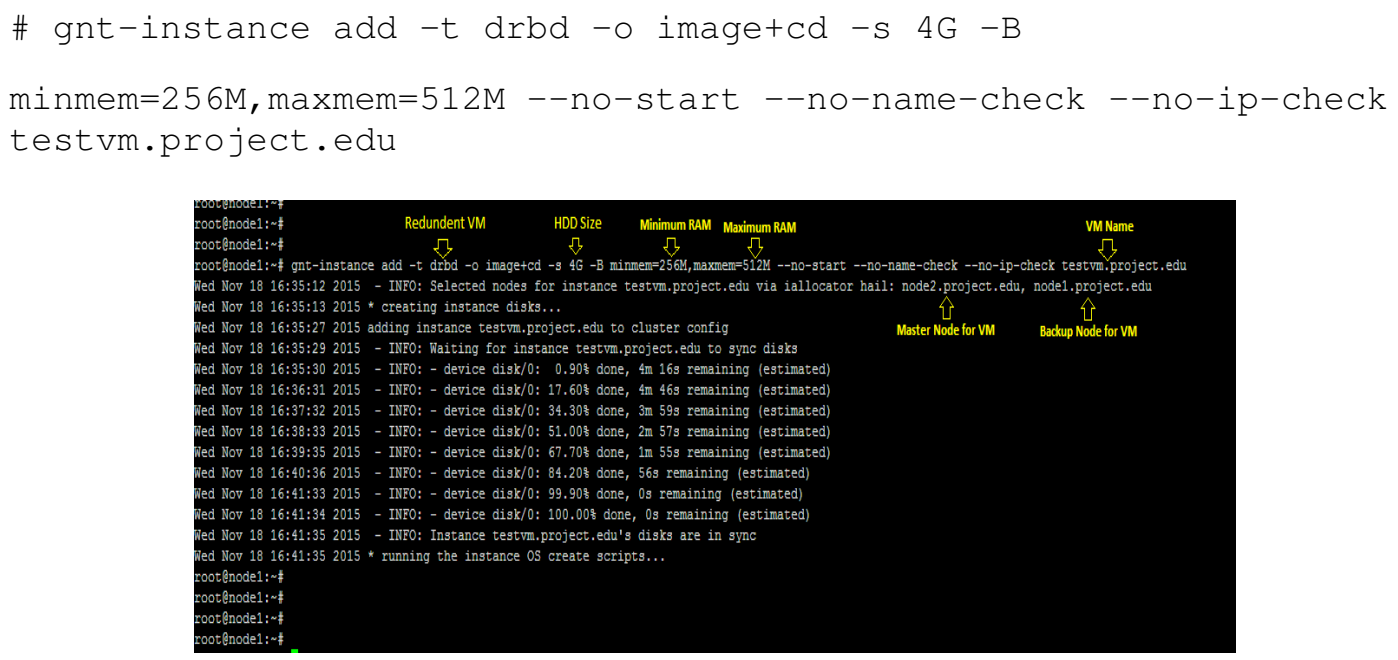

Figure 2.13: Instance create 


\section{Explanation:}

-t drbd means replicated LVM (replicated with DRBD)

-o image+cd means to use the OS definition ganeti-instance-image, with the cd variant we created -s 4G means to create a 4GB disk drive

-B minmem=256M,maxmem $=512 \mathrm{M}$ sets the memory limits for this VM. It will try to run it with $512 \mathrm{M}$, but if not enough memory is available it may shrink it down to 256MB.

--no-start means don't start the VM after creating it

--no-name-check means don't check that testvm.project.edu exists in the DNS (because it doesn't!)

--no-ip-check means if you found the name in the DNS, don't check that the IP address is not in usethe final parameter is the name of the instance. It would be good practice to use a fullyqualified domain name for this.

You will see some messages about creating the instance being created.

\section{RESULTS}

\subsection{RUN AN INSTANCE}

Now start the VM using the following command, which attaches the CD-ROM temporarily and boots from it:

\# gnt-instance start $-\mathrm{H}$

boot_order=cdrom, cdrom_image_path=/iso/debian-7.9.0-amd64-

netinst.iso testvm.project.edu

Waiting for job 332 for testvm.project.edu ...

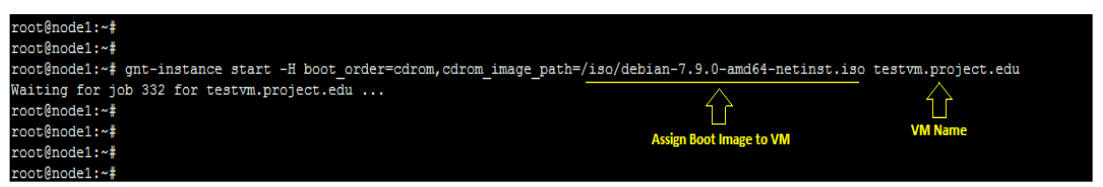

Figure 3.1: Run an instance

\subsection{Verify the configuration of your cluster}

Again only on the MASTER node of the cluster:

\# gnt-cluster verify

This will tell you if there are any errors in your configuration.

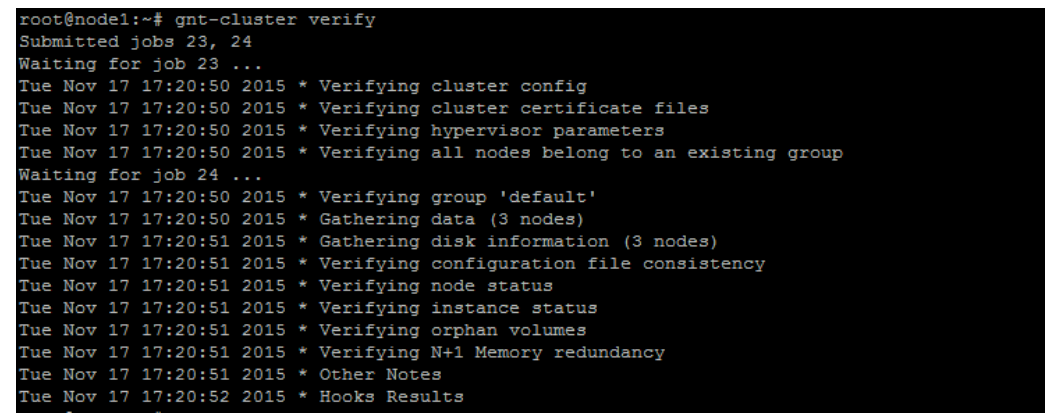

Figure 3.2: Verify Cluster 


\subsection{CHECK DETAIL InFormation About An InSTANCE}

Ganeti will assign a port for console access for the created VM so that we can install the operating system on it remotely. Here is how to check it.

\# gnt-instance info testvm.project.edu

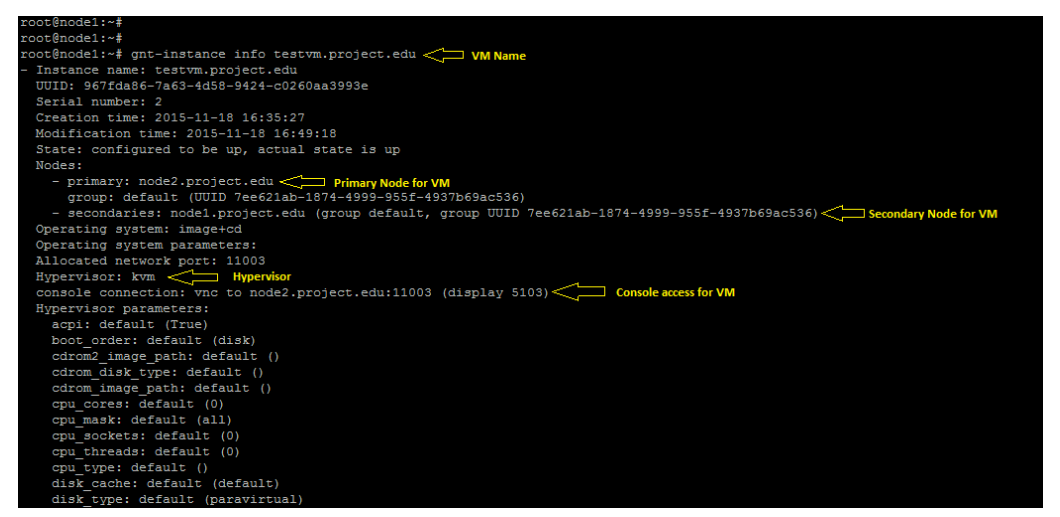

Figure 3.3: Information about an instance-1

More information about testvm.project.edu

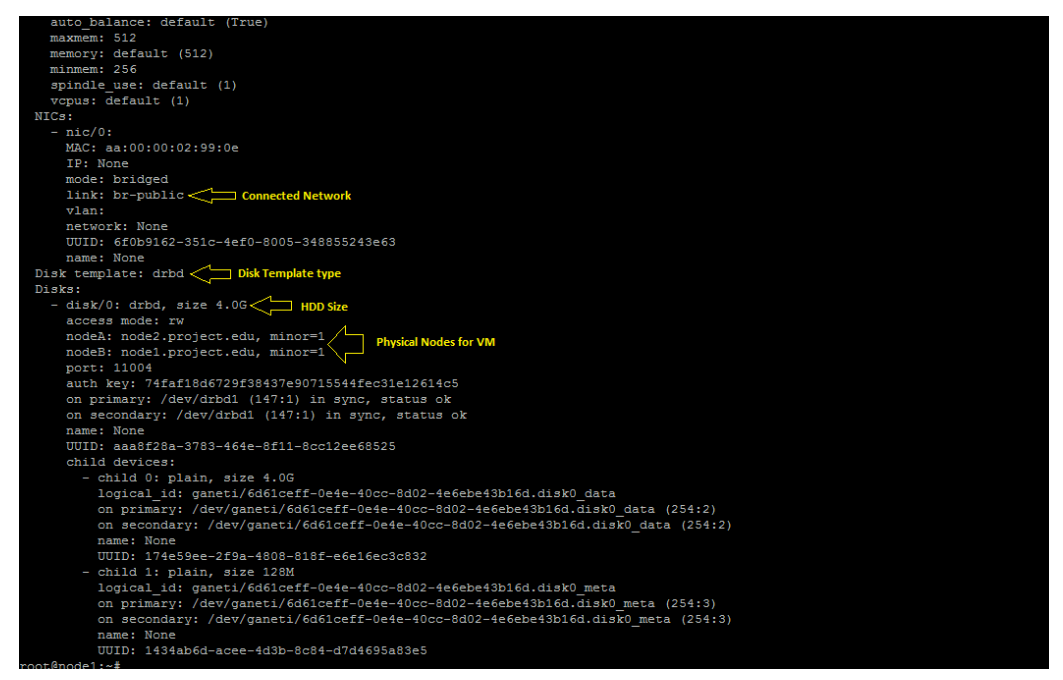

Figure 3.4: Information about an instance-2

\subsection{InStall A Guest Operating System In An InStance}

We can see the console access for the VM is node2.project.edu:11003. We will use a VNC viewer to access the VM and install the operating system on it. 
International Journal on Cloud Computing: Services and Architecture (IJCCSA) Vol. 6, No. 2, April 2016

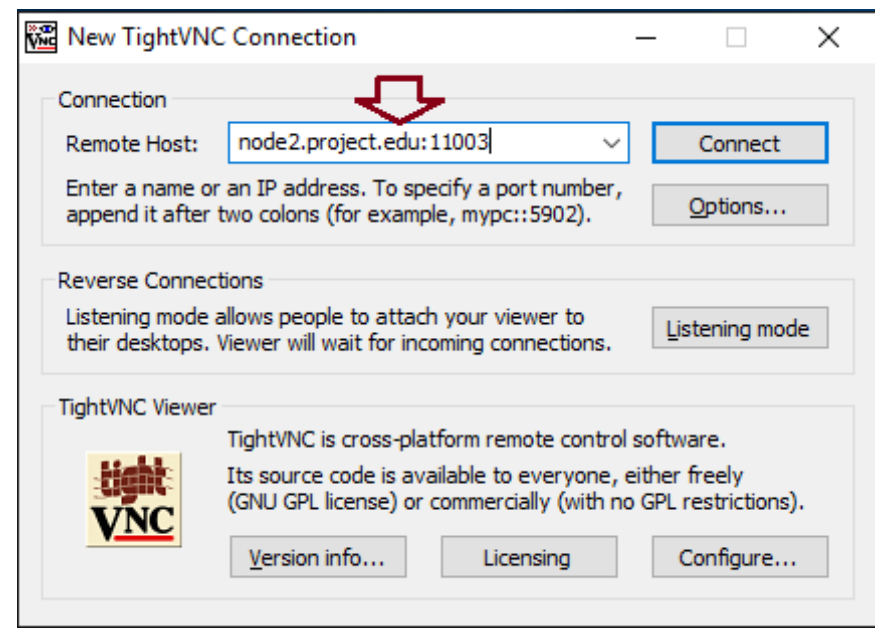

Figure 3.5: Connect an instance by VNC Viewer

By clicking "Connect" button the console will appear in front of us and we will install the OS on testvm.project. edu instance with the IP address of 192.168.20.232.

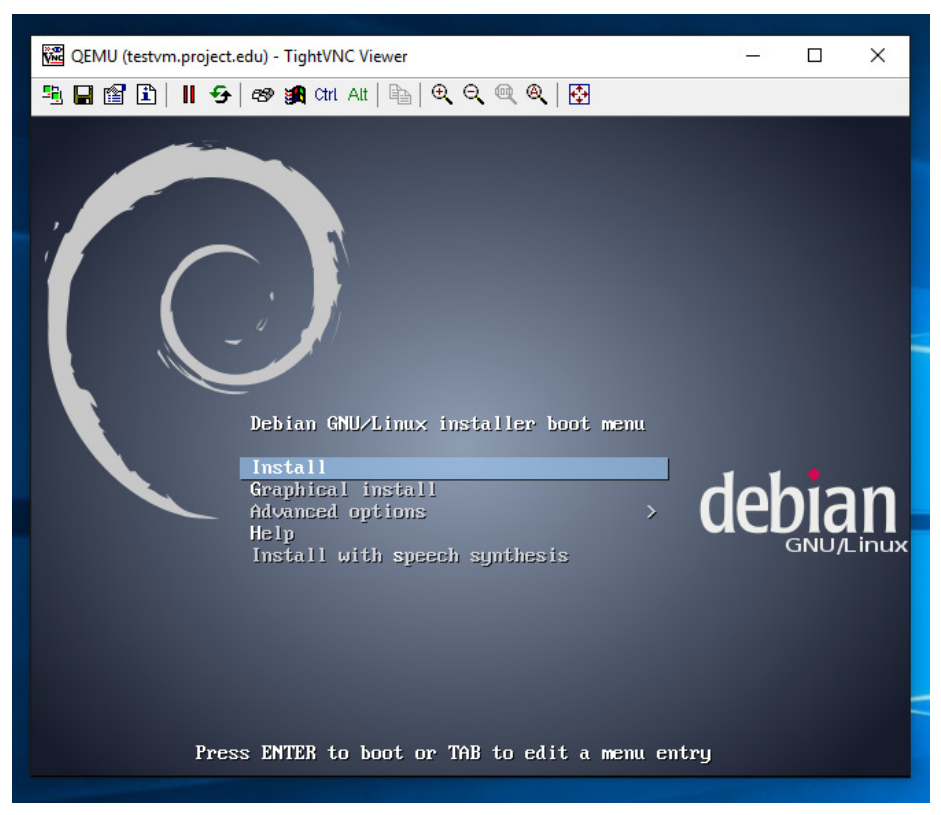

Figure 3.6: Install Guest Operating System in an Instance

\subsection{Changing Network Of An InStance}

We may not need to run this, but if we want to we can. Let's say we have informed our cluster "br-public" as the default network for every instance. Now we are connected to the "br-man" network. As a result we cannot access the VM from remote network as "br-man" is not published to internet. 
International Journal on Cloud Computing: Services and Architecture (IJCCSA) Vol. 6, No. 2, April 2016

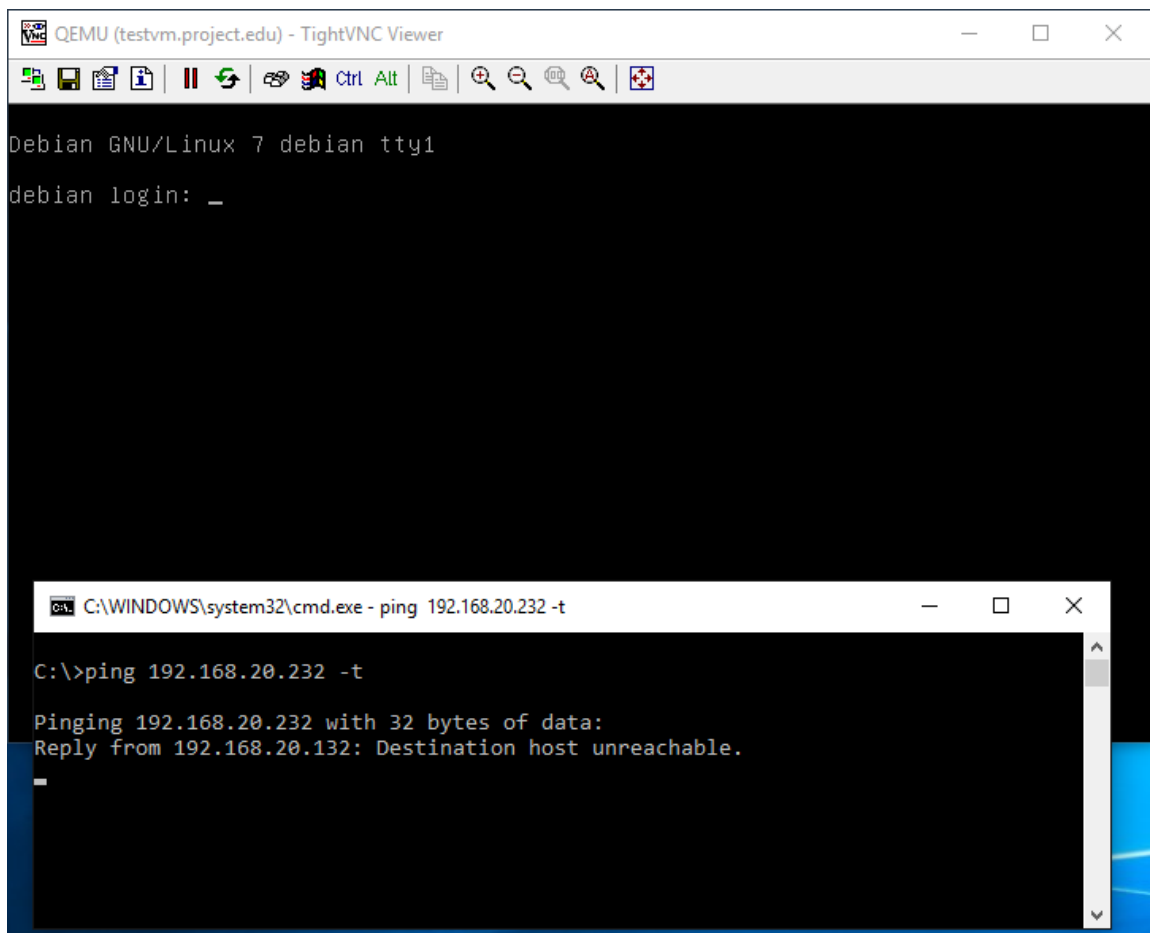

Figure 3.7: Connectivity check for an instance

Now we need to change the network of the instance to "br-man" from "br-public". Here is how to do that:

Moving the network interface 0 to another network:

\# gnt-instance modify --net 0:modify, link=br-man --hotplug testvm.project.edu

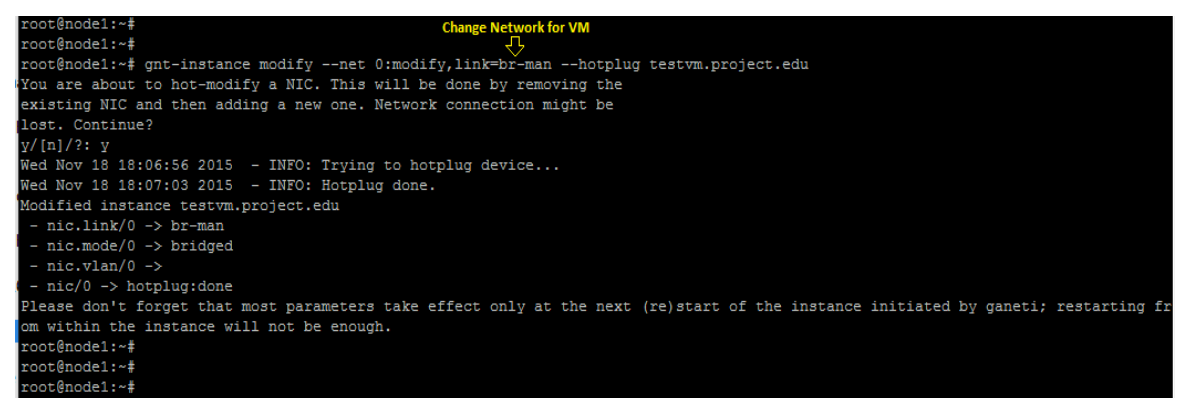

Figure 3.8: Change the network for an instance

Try to do this to move the network interface of one of the instances you created earlier, onto the br-man. After successfully shifting the network, now we can access the instance without any problem. 
International Journal on Cloud Computing: Services and Architecture (IJCCSA) Vol. 6, No. 2, April 2016

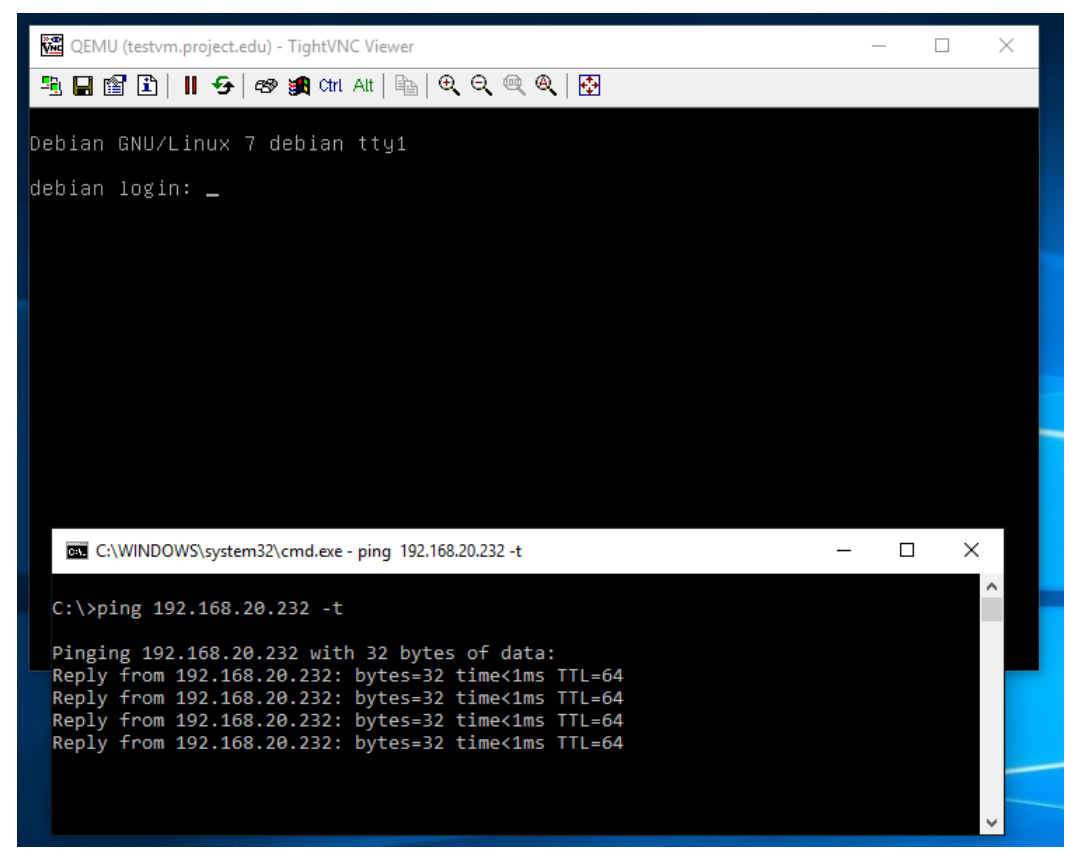

Figure 3.9: Check availability after changing the network of an instance

\subsection{Testing Service Migration Between Two InstanceS}

In some cases we may need to change the service of an instance to backup node. Let's say we have an instance running with DRBD replication on node2 . pro ject . edu as primary node and node1.project.edu as backup node. Now we have a situation and we need to shut down node2.project.edu for maintenance. We should do it without interrupting the users from their service. So we will be migrated the service to node1.project.edu and shut down node2.project.edu for maintenance.

\# gnt-instance migrate testvm.project.edu

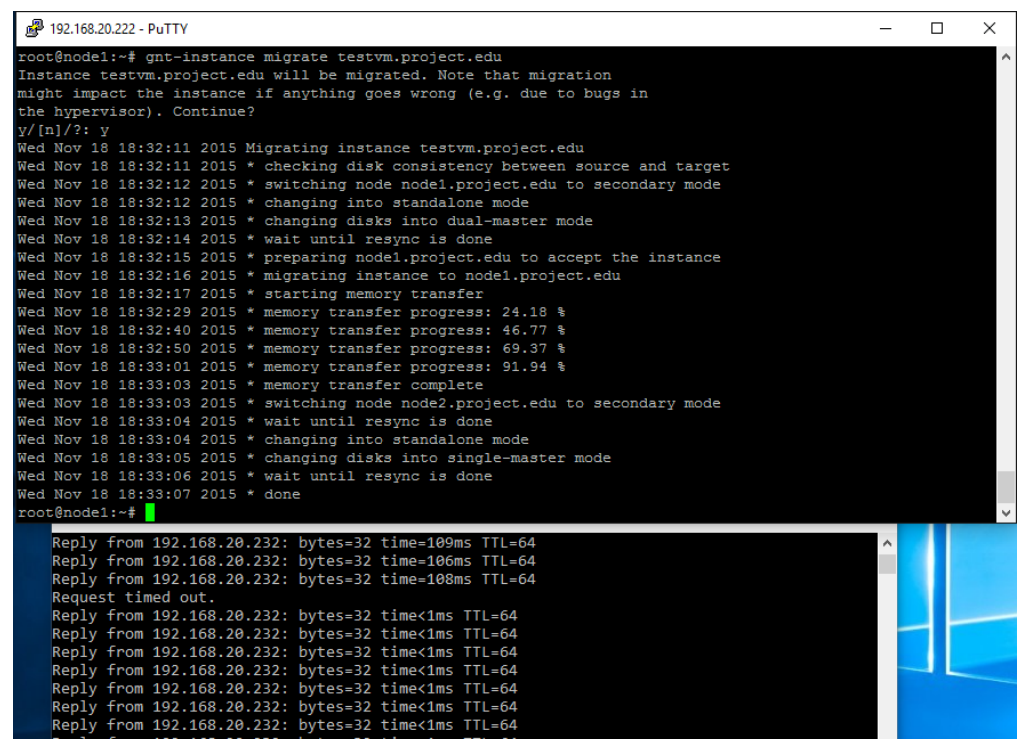

Figure 3.10: Live service migration of an instance 


\subsection{INSTANCE FAILOVER SCENARIO}

Suppose we have an instance running on node 2. project.edu as its primary node and node1.project.edu as its backup node. Suddenly disaster happens; node2.project.edu has failed and went down.

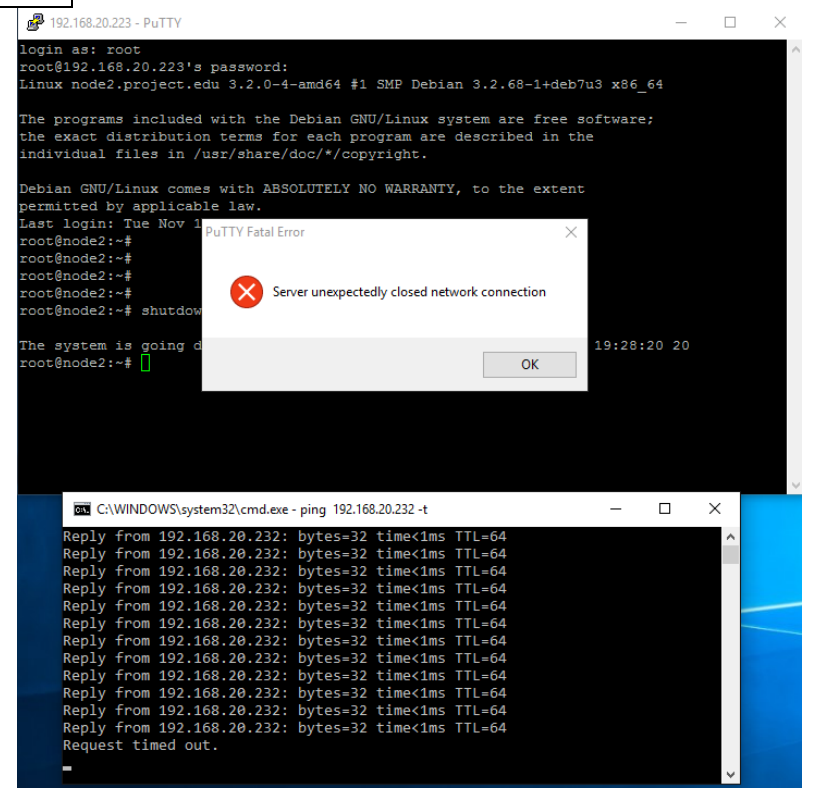

Figure 3.11: Failover of an instance

Instances' running on that node has been down and services stopped. But we can make the service alive within short time without losing any data of that instance by following command.

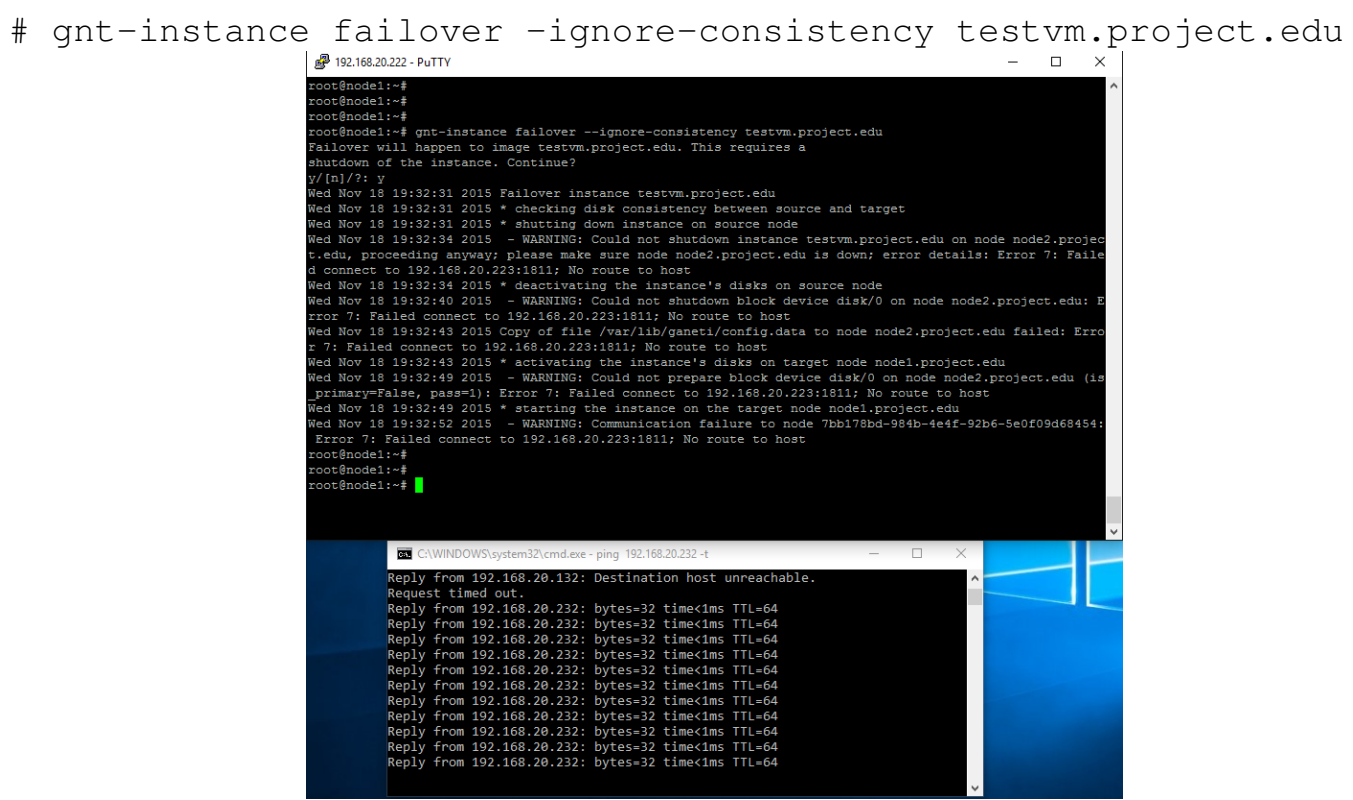

Figure 3.12: Recovery of an instance after failure 
Now if we check the instance list we can see the instance is running on node1.project.edu as its primary node.

\# gnt-instance list -o name, pnode, snodes, status

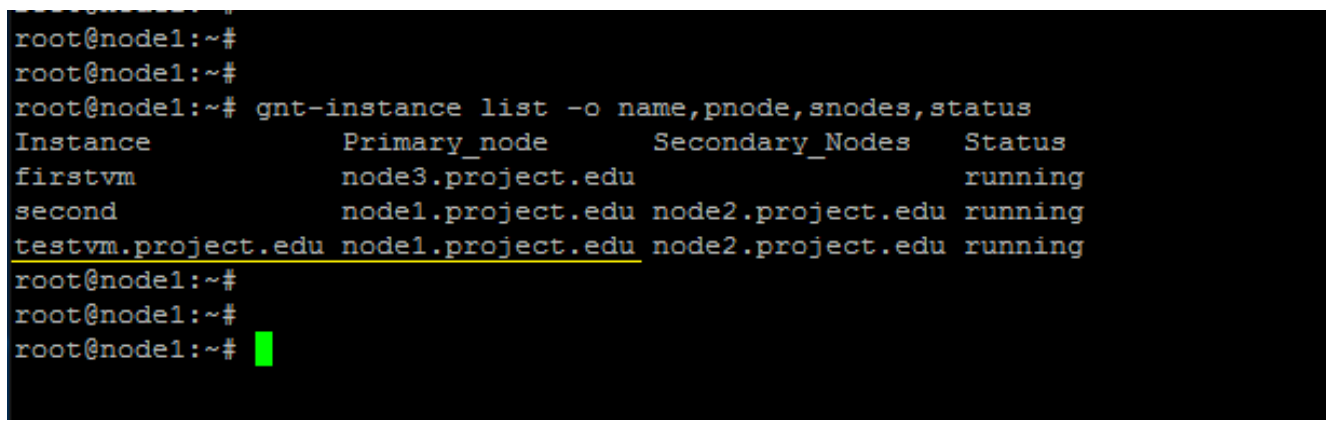

Figure 3.13: Check total instances of the Cluster

\section{CONCLUSION AND Future WORK}

In our project we have tried our best to run the virtualization over commodity hardware and create some VMs on it. We have successfully completed the job. Later we tried to introduce some scenarios and recommended some standard way-out of those cases. We can suggest this project for small and medium office if they want to move for virtualization of their services using existing commodity hardware.

Probing deeper, one can use a web management tool for Ganeti administration. Moreover if the cluster used for business and provided SaaS to the customers, one can work on the development of a web interface for system administrators so that they can manage and check billing of their uses which will be a very useful tool for provider as well as customer.

Table 1. Heading and text fonts.

\begin{tabular}{|l|l|l|l|}
\hline Text & Alignment & Font & Followed by: \\
\hline Title & Centre & 20 pt. TNR, bold, small-caps & 24 pt. line sp. \\
\hline Authors & Centre & 13 pt. TNR & 12 pt. line sp. \\
\hline Addresses & Centre & 12 pt. TNR & \\
\hline emails & Centre & 11 pt. italic TNR & 18 pt. line sp. (last) \\
\hline Abstract heading & Left & 13 pt. bold italic TNR, small caps & 6 pt. line sp. \\
\hline Abstract text & Left & 10 pt. italic TNR & 12 pt. line sp. \\
\hline Keywords heading & Left & 13 pt. bold italic TNR, small caps & 6 pt. line sp. \\
\hline Keywords & Left, left, .. & 10 pt. italic TNR & 18 pt line sp. \\
\hline Section headings & Left & 14 pt. bold TNR, small caps & 6 pt. line sp. \\
\hline Sub-section heads & Left & 12 pt. bold TNR & 6 pt. line sp. \\
\hline Sub-sub-sections & Left & 11 pt. bold TNR & 6 pt. line sp. \\
\hline Body text & Full (left/right) & 11 pt. TNR & 12 pt line sp. (last) \\
\hline Figures & Centre & & 6 pt. line sp. \\
\hline Figure captions & Centre & 11 pt. TNR & 12 pt. line sp. \\
\hline References & Left & 10 pt. TNR (as shown) & 6 pt. line sp \\
\hline
\end{tabular}




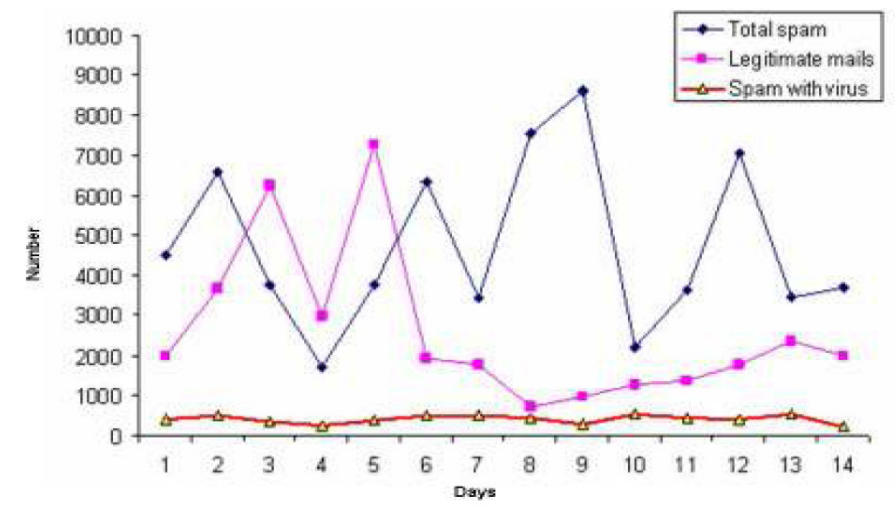

\section{REFERENCES}

Figure 4. Spam traffic sample

[1 ]Mollah, Muhammad Baqer, Kazi Raisul Islam, and Sikder Sunbeam Islam. "Next generation of computing through cloud computing technology." Electrical \& Computer Engineering (CCECE), 2012 25th IEEE Canadian Conference on. IEEE, 2012

[2] Soltesz, S., Pötzl, H., Fiuczynski, M. E., Bavier, A., \& Peterson, L. (2007, March). Container-based operating system virtualization: a scalable, high-performance alternative to hypervisors. In ACM SIGOPS Operating Systems Review (Vol. 41, No. 3, pp. 275-287). ACM.

[3] Padala, P., Zhu, X., Wang, Z., Singhal, S., \& Shin, K. G. (2007). Performance evaluation of virtualization technologies for server consolidation. HP Labs Tec. Report.

[4] Regola, N., \& Ducom, J. C. (2010, November). Recommendations for virtualization technologies in high performance computing. In Cloud Computing Technology and Science (CloudCom), 2010 IEEE Second International Conference on (pp. 409-416). IEEE.

[5] Sharma, S. (2016). Expanded cloud plumes hiding Big Data ecosystem. Future Generation Computer Systems, 59, 63-92.

\section{Authors}

Mohammad Mamun Or Rashid received his B.Sc. (Hon's) in Computer Science from North South University (NSU), Dhaka, Bangladesh in 2006 and M.Sc. in Computer Science in 2015 from Jahangirnagar University, Savar, Dhaka, Bangladesh. He has been working in Government of the People's Republic of Bangladesh as a "System Analyst" in Ministry of Expatriate's Welfare and Overseas Employment. His current research interests include Cloud Computing, virtualization and information Security management system. He is also interested in Linux and Virtual networking in cloud computing.

M. Masud Rana received the B.Sc. in Computer Science and Engineering from the Dhaka International University, Dhaka, Bangladesh in 2014. Currently, he is working towards M.Sc. in Computer Science from the Jahangirnagar University, Savar, Dhaka, Bangladesh. He has serving as an Executive Engineer, Information Technology in Bashundhara Group also he has more than 5 years of experience as an Assistant Engineer, IT in SQUARE Informatix Ltd, Bangladesh and Executive Engineer, IT in Computer Source Ltd, Bangladesh. His main areas of research interests include virtualization, networking and security aspects of cloud

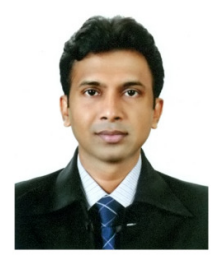
computing

Jugal Krishna Das received B.Sc., M.Sc. and PhD in Computer Science all from Rassia. He is currently an Professor of Computer Science and Engineering department of Jahangirnagar University, Savar, Dhaka. His research interests include topics such as Computer Networks, Natural Language Processing, Software Engineering.
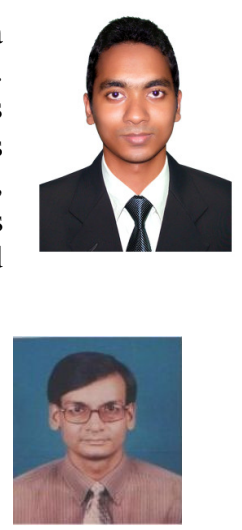